\title{
STRONG CONVERGENCE THEOREM OF CESÀRO MEANS WITH RESPECT TO THE WALSH SYSTEM
}

\author{
ISTVÁN BLAHOTA, GIORGI TEPHNADZE AND RODOLFO TOLEDO
}

\begin{abstract}
We prove that Cesàro means of one-dimensional WalshFourier series are uniformly bounded operators in the martingale Hardy space $H_{p}$ for $0<p<1 /(1+\alpha)$.
\end{abstract}

\section{INTRODUCTION}

The definitions and notations used in this introduction can be found in the next Section. It is well-known (see, e.g., [11, p.125]) that Walsh-Paley system is not a Schauder basis in the space $L_{1}(G)$. Moreover, there is a function $F$ in the dyadic Hardy space $H_{1}(G)$, such that the partial sums of the Walsh-Fourier series of $F$ are not bounded in the $L_{1}$-norm. However, in Simon [19] the following estimation was obtained: for all $F \in H_{1}(G)$

$$
\frac{1}{\log n} \sum_{k=1}^{n} \frac{\left\|S_{k} F\right\|_{1}}{k} \leq c\|F\|_{H_{1}}, \quad(n=2,3, \ldots),
$$

where $S_{k} F$ denotes the $k$-th partial sum of the Walsh-Fourier series of $F$ (For the trigonometric analogue see in Smith [21], for the Vilenkin system in Gát [6], for a more general, so-called Vilenkin-like system in Blahota [1]). Simon [16] (see also [27] and [34]) proved that there exists an absolute constant $c_{p}$, depending only on $p$, such that

$$
\frac{1}{\log ^{[p]} n} \sum_{k=1}^{n} \frac{\left\|S_{k} F\right\|_{p}^{p}}{k^{2-p}} \leq c_{p}\|F\|_{H_{p}}^{p}, \quad(0<p \leq 1, n=2,3, \ldots),
$$

for all $F \in H_{p}$, where $[p]$ denotes integer part of $p$.

In [25] it was proven that sequence $\left\{1 / k^{2-p}\right\}_{k=1}^{\infty}(0<p<1)$ in (11) is given exactly.

Weisz [35] considered the norm convergence of Fejér means of WalshFourier series and proved that

$$
\left\|\sigma_{n} F\right\|_{H_{p}} \leq c_{p}\|F\|_{H_{p}}, \quad F \in H_{p}, \quad(1 / 2<p<\infty, \quad n=1,2,3, \ldots),
$$

where the constant $c_{p}>0$ depends only on $p$.

2010 Mathematics Subject Classification. 42C10.

Key words and phrases. Walsh system, Cesàro mean, martingale Hardy space.

The research was supported by project TÁMOP-4.2.2.A-11/1/KONV-2012-0051 and by Shota Rustaveli National Science Foundation grant no.52/54 (Bounded operators on the martingale Hardy spaces). 
Inequality (2) immediately implies that

$$
\frac{1}{n^{2 p-1}} \sum_{k=1}^{n} \frac{\left\|\sigma_{k} F\right\|_{H_{p}}^{p}}{k^{2-2 p}} \leq c_{p}\|F\|_{H_{p}}^{p}, \quad(1 / 2<p<\infty) .
$$

If (2) also hold, for $0<p \leq 1 / 2$, then we would have

$$
\frac{1}{\log ^{[1 / 2+p]} n} \sum_{k=1}^{n} \frac{\left\|\sigma_{k} F\right\|_{H_{p}}^{p}}{k^{2-2 p}} \leq c_{p}\|F\|_{H_{p}}^{p}, \quad(0<p \leq 1 / 2, n=2,3, \ldots),
$$

but in 22$]$ it was proven that the assumption $p>1 / 2$ is essential. In particular, there was proven that there exists a martingale $F \in H_{p}(0<p \leq 1 / 2)$, such that $\sup _{n}\left\|\sigma_{n} F\right\|_{p}=+\infty$.

However, in [26] (see also [3]) it was proven that (3) holds, though (2) is not true for $0<p \leq 1 / 2$.

The weak-type $(1,1)$ inequality for the maximal operator of Fejér means $\sigma^{*}$ can be found in Schipp [14] (see also [13]). Fujji [5] and Simon [18] verified that $\sigma^{*}$ is bounded from $H_{1}$ to $L_{1}$. Weisz [30] generalized this result and proved the boundedness of $\sigma^{*}$ from the space $H_{p}$ to the space $L_{p}$ for $p>1 / 2$. Simon 17] gave a counterexample, which shows that boundedness does not hold for $0<p<1 / 2$. The counterexample for $p=1 / 2$ is due to Goginava [8] (see also [4]). Weisz [31] proved that $\sigma^{*}$ is bounded from the Hardy space $H_{1 / 2}$ to the space $L_{1 / 2, \infty}$. In [23, 24] it was proven that the maximal operators $\widetilde{\sigma}_{p}^{*}$ defined by

$$
\widetilde{\sigma}_{p}^{*} F:=\sup _{n \in \mathbb{N}} \frac{\left|\sigma_{n} F\right|}{n^{1 / p-2} \log ^{2[1 / 2+p]} n}, \quad(0<p \leq 1 / 2, n=2,3, \ldots)
$$

is bounded from the Hardy space $H_{p}$ to the space $L_{p}$, where $F \in H_{p}$ and $[1 / 2+p]$ denotes integer part of $1 / 2+p$. Moreover, there was also shown that sequence $\left\{n^{1 / p-2} \log ^{2[1 / 2+p]} n: n=2,3, \ldots\right\}$ in (44) can not be improved.

Weisz [33] proved that the maximal operator $\sigma^{\alpha, *}(0<\alpha<1)$ of the Cesàro means of Walsh system is bounded from the martingale space $H_{p}$ to the space $L_{p}$ for $p>1 /(1+\alpha)$. Goginava [9] gave a counterexample, which shows that the boundedness does not hold for $0<p \leq 1 /(1+\alpha)$. Recently, Weisz and Simon [20] show that the maximal operator $\sigma^{\alpha, *}$ is bounded from the Hardy space $H_{1 /(1+\alpha)}$ to the space $L_{1 /(1+\alpha), \infty}$. An analogical result for Walsh-Kaczmarz system was proven in [7].

In 10] Goginava investigated the behaviour of Cesàro means of WalshFourier series in detail. For some approximation properties of the two dimensional case see paper of Nagy [12].

The main aim of this paper is to generalize estimate (3) for Cesàro means, when $0<p<1 /(1+\alpha)$. We also consider the weighted maximal operator of $(C, \alpha)$ means and proved some new $\left(H_{p}, L_{p}\right)$-type inequalities for it.

We note that the case $p=1 /(1+\alpha)$ was considered in [2]. 


\section{Definitions and Notations}

Let $\mathbb{N}_{+}$denote the set of the positive integers, $\mathbb{N}:=\mathbb{N}_{+} \cup\{0\}$. Denote by $\mathbb{Z}_{2}$ the discrete cyclic group of order 2 , that is $\mathbb{Z}_{2}:=\{0,1\}$, where the group operation is the modulo 2 addition and every subset is open. The Haar measure on $\mathbb{Z}_{2}$ is given so that the measure of a singleton is $1 / 2$.

Define the group $G$ as the complete direct product of the group $\mathbb{Z}_{2}$ with the product of the discrete topologies of $\mathbb{Z}_{2}$ 's. The elements of $G$ are represented by sequences

$$
x:=\left(x_{0}, x_{1}, \ldots, x_{k}, \ldots\right) \quad\left(x_{k}=0,1\right) .
$$

It is easy to give a base for the neighborhood of $G$

$$
\begin{aligned}
I_{0}(x): & =G \\
I_{n}(x) & :=\left\{y \in G \mid y_{0}=x_{0}, \ldots, y_{n-1}=x_{n-1}\right\}(x \in G, n \in \mathbb{N}) .
\end{aligned}
$$

Denote $I_{n}:=I_{n}(0)$ and $\overline{I_{n}}:=G \backslash I_{n}$. Let

$$
e_{n}:=\left(0, \ldots, 0, x_{n}=1,0, \ldots\right) \in G \quad(n \in \mathbb{N})
$$

Denote

$I_{M}^{k, l}:= \begin{cases}I_{M}\left(0, \ldots, 0, x_{k}=1,0, \ldots, 0, x_{l}=1, x_{l+1}, \ldots, x_{M-1}\right), & k<l<M, \\ I_{M}\left(0, \ldots, 0, x_{k}=1,0, \ldots, 0\right), & l=M .\end{cases}$

It is evident

$$
\overline{I_{M}}=\left(\bigcup_{k=0}^{M-2} \bigcup_{l=k+1}^{M-1} I_{M}^{k, l}\right) \bigcup\left(\bigcup_{k=0}^{M-1} I_{M}^{k, M}\right) .
$$

If $n \in \mathbb{N}$, then every $n$ can be uniquely expressed as $n=\sum_{j=0}^{\infty} n_{j} 2^{j}$, where $n_{j} \in Z_{2}(j \in \mathbb{N})$ and only finite number of $n_{j}$ 's differ from zero, that is, $n$ is expressed in the number system of base 2 . Let $|n|:=\max \left\{j \in \mathbb{N}, n_{j} \neq 0\right\}$, that is $2^{|n|} \leq n \leq 2^{|n|+1}$.

The norm (or quasi-norm) of the space $L_{p}(G)$ is defined by

$$
\|f\|_{p}:=\left(\int_{G}|f|^{p} d \mu\right)^{1 / p},(0<p<\infty) .
$$

The space $L_{p, \infty}(G)$ consists of all measurable functions $f$, for which

$$
\|f\|_{L_{p, \infty}(G)}:=\sup _{\lambda>0} \lambda \mu(f>\lambda)^{1 / p}<\infty .
$$

Next, we introduce on $G$ an orthonormal system which is called the Walsh system. At first, define the functions $r_{k}(x): G \rightarrow \mathbb{C}$, the so-called Rademacher functions as

$$
r_{k}(x):=(-1)^{x_{k}} \quad(x \in G, k \in \mathbb{N}) .
$$

Now, define the Walsh system $w:=\left(w_{n}: n \in \mathbb{N}\right)$ on $G$ as:

$$
w_{n}(x):=\prod_{k=0}^{\infty} r_{k}^{n_{k}}(x)=r_{|n|}(x)(-1) \sum_{k=0}^{|n|-1} n_{k} x_{k} \quad(n \in \mathbb{N}) .
$$


The Walsh system is orthonormal and complete in $L_{2}(G)$ (see, e.g., [28]).

If $f \in L_{1}(G)$, then we can establish Fourier coefficients, partial sums of the Fourier series, Fejér means, Dirichlet and Fejér kernels in the usual manner:

$$
\begin{array}{rlrl}
\widehat{f}(n):=\int_{G} f w_{n} d \mu, & & (n \in \mathbb{N}), \\
S_{n} f:=\sum_{k=0}^{n-1} \widehat{f}(k) w_{k}, & & \left(n \in \mathbb{N}_{+}\right), \\
\sigma_{n} f:=\frac{1}{n} \sum_{k=1}^{n} S_{k} f, & \left(n \in \mathbb{N}_{+}\right), \\
D_{n}:=\sum_{k=0}^{n-1} w_{k}, & \left(n \in \mathbb{N}_{+}\right), \\
K_{n}:=\frac{1}{n} \sum_{k=1}^{n} D_{k}, & \left(n \in \mathbb{N}_{+}\right),
\end{array}
$$

respectively. Recall that (see e.g., [15])

$$
D_{2^{n}}(x)= \begin{cases}2^{n}, & \text { if } x \in I_{n}, \\ 0, & \text { if } x \notin I_{n} .\end{cases}
$$

The Cesàro means $((C, \alpha)$-means) are defined as

$$
\sigma_{n}^{\alpha} f:=\frac{1}{A_{n}^{\alpha}} \sum_{k=1}^{n} A_{n-k}^{\alpha-1} S_{k} f,
$$

where

$$
A_{0}^{\alpha}:=1, \quad A_{n}^{\alpha}:=\frac{(\alpha+1) \ldots(\alpha+n)}{n !} \quad \alpha \neq-1,-2, \ldots
$$

It is well known that

$$
A_{n}^{\alpha}=\sum_{k=0}^{n} A_{n-k}^{\alpha-1}, A_{n}^{\alpha}-A_{n-1}^{\alpha}=A_{n}^{\alpha-1}, A_{n}^{\alpha} \backsim n^{\alpha}
$$

and

$$
\sup _{n} \int_{G}\left|K_{n}^{\alpha}\right| d \mu \leq c<\infty
$$

where $K_{n}^{\alpha}$ is $n$th Cesàro kernel.

The $\sigma$-algebra generated by the intervals $\left\{I_{n}(x): x \in G\right\}$ will be denoted by $F_{n}(n \in \mathbb{N})$. Denote by $F=\left(F_{n}, n \in \mathbb{N}\right)$ the martingale with respect to $F_{n}(n \in \mathbb{N})$ (for details see, e.g., [29]).

The maximal function of a martingale $F$ is defined by

$$
F^{*}:=\sup _{n \in \mathbb{N}}\left|F_{n}\right|
$$


In the case $f \in L_{1}(G)$, the maximal functions are also be given by

$$
f^{*}(x)=\sup _{n \in \mathbb{N}} \frac{1}{\mu\left(I_{n}(x)\right)}\left|\int_{I_{n}(x)} f(u) d \mu(u)\right| .
$$

For $0<p<\infty$, the Hardy martingale spaces $H_{p}(G)$ consist of all martingales such that

$$
\|F\|_{H_{p}}:=\left\|F^{*}\right\|_{p}<\infty
$$

A bounded measurable function $a$ is a $p$-atom, if there exist a dyadic interval $I$ such that

$$
\int_{I} a d \mu=0, \quad\|a\|_{\infty} \leq \mu(I)^{-1 / p}, \quad \operatorname{supp}(a) \subset I .
$$

It is easy to check that for every martingale $F=\left(F_{n}, n \in \mathbb{N}\right)$ and for every $k \in \mathbb{N}$ the limit

$$
\widehat{F}(k):=\lim _{n \rightarrow \infty} \int_{G} F_{n} w_{k} d \mu
$$

exists and it is called the $k$-th Walsh-Fourier coefficients of $F$.

Denote by $\mathcal{A}_{n}$ the $\sigma$-algebra generated by the sets $I_{n}(x)(x \in G, n \in \mathbb{N})$. If $F:=\left(S_{2^{n}} f: n \in \mathbb{N}\right)$ is the regular martingale generated by $f \in L_{1}(G)$, then

$$
\widehat{F}(k)=\int_{G} f w_{k} d \mu=: \widehat{f}(k), \quad k \in \mathbb{N} .
$$

For $0<\alpha \leq 1$, let consider maximal operators

$$
\sigma^{\alpha, *} F:=\sup _{n \in \mathbb{N}}\left|\sigma_{n}^{\alpha} F\right|, \quad \tilde{\sigma}_{p}^{\alpha, *} F:=\sup _{n \in \mathbb{N}} \frac{\left|\sigma_{n}^{\alpha} F\right|}{(n+1)^{1 / p-1-\alpha}}, 0<p<1 /(1+\alpha) .
$$

For the martingale

$$
F=\sum_{n=0}^{\infty}\left(F_{n}-F_{n-1}\right)
$$

the conjugate transforms are defined as

$$
\widetilde{F^{(t)}}:=\sum_{n=0}^{\infty} r_{n}(t)\left(F_{n}-F_{n-1}\right)
$$

where $t \in G$ is fixed. Note that $\widetilde{F^{(0)}}=F$.

As it is well-known (see, e.g., [29])

$$
\left\|\widetilde{F^{(t)}}\right\|_{H_{p}}=\|F\|_{H_{p}}, \quad\|F\|_{H_{p}}^{p} \sim \int_{G}\left\|\widetilde{F^{(t)}}\right\|_{p}^{p} d t, \quad \widetilde{\sigma_{m}^{\alpha} F^{(t)}}=\sigma_{m}^{\alpha} \widetilde{F^{(t)}} .
$$




\section{Formulation of MAin Results}

Theorem 1. a) Let $0<\alpha<1$ and $0<p<1 /(1+\alpha)$. Then there exists absolute constant $c_{\alpha, p}$, depending on $\alpha$ and $p$, such that for all $F \in H_{p}(G)$

$$
\left\|\tilde{\sigma}_{p}^{\alpha, *} F\right\|_{p} \leq c_{\alpha, p}\|F\|_{H_{p}}
$$

b) Let $0<\alpha<1,0<p<1 /(1+\alpha)$ and $\varphi: \mathbb{N}_{+} \rightarrow[1, \infty)$ be a nondecreasing function satisfying the condition

$$
\varlimsup_{n \rightarrow \infty} \frac{n^{1 / p-1-\alpha}}{\varphi(n)}=\infty .
$$

Then the maximal operator

$$
\sup _{n \in \mathbb{N}} \frac{\left|\sigma_{n}^{\alpha} f\right|}{\varphi(n)}
$$

is not bounded from the Hardy space $H_{p}(G)$ to the space $L_{p}(G)$.

Theorem 2. Let $0<\alpha<1$ and $0<p<1 /(1+\alpha)$. Then there exists absolute constant $c_{\alpha, p}$, depending on $\alpha$ and $p$, such that for all $F \in H_{p}$

$$
\sum_{m=1}^{\infty} \frac{\left\|\sigma_{m}^{\alpha} F\right\|_{H_{p}}^{p}}{m^{2-(1+\alpha) p}} \leq c_{\alpha, p}\|F\|_{H_{p}}^{p} .
$$

\section{Auxiliary Propositions}

The dyadic Hardy martingale spaces $H_{p}(G)$ have an atomic characterization, when $0<p \leq 1$ :

Lemma 1. (Weisz [32] A martingale $F=\left(F_{n}, n \in \mathbb{N}\right)$ is in $H_{p}(0<p \leq 1)$ if and only if there exists a sequence $\left(a_{k}, k \in \mathbb{N}\right)$ of $p$-atoms and a sequence $\left(\mu_{k}, k \in \mathbb{N}\right)$ of a real numbers, such that for every $n \in \mathbb{N}$

$$
\begin{aligned}
& \sum_{k=0}^{\infty} \mu_{k} S_{2^{n}} a_{k}=F_{n}, \\
& \sum_{k=0}^{\infty}\left|\mu_{k}\right|^{p}<\infty .
\end{aligned}
$$

Moreover,

$$
\|F\|_{H_{p}} \backsim \inf \left(\sum_{k=0}^{\infty}\left|\mu_{k}\right|^{p}\right)^{1 / p},
$$

where the infimum is taken over all decompositions of $F$ of the form (12).

By using Lemma 1 we can easily proved the following: 
Lemma 2. (Weisz [29]) Suppose that an operator $T$ is $\sigma$-linear and for some $0<p \leq 1$

$$
\int_{\bar{I}}|T a|^{p} d \mu \leq c_{p}<\infty,
$$

for every p-atom a, where I denote the support of the atom. If $T$ is bounded from $L_{\infty}$ to $L_{\infty}$, then

$$
\|T f\|_{p} \leq c_{p}\|f\|_{H_{p}} .
$$

To prove our main results we also need the following estimations:

Lemma 3. 2] Let $0<\alpha<1$ and $n>2^{M}$. Then

$$
\int_{I_{M}}\left|K_{n}^{\alpha}(x+t)\right| d \mu(t) \leq \frac{c_{\alpha} 2^{\alpha l+k}}{n^{\alpha} 2^{M}}
$$

for $x \in I_{l+1}\left(e_{k}+e_{l}\right),(k=0, \ldots, M-2, l=k+1, \ldots, M-1)$ and

$$
\int_{I_{M}}\left|K_{n}^{\alpha}(x+t)\right| d \mu(t) \leq \frac{c_{\alpha} 2^{k}}{2^{M}},
$$

for $x \in I_{M}\left(e_{k}\right),(k=0, \ldots, M-1)$.

\section{Proof of Theorems}

Proof of Theorem 1. Since $\sigma_{n}$ is bounded from $L_{\infty}$ to $L_{\infty}$ (the boundedness follows from (8) ) according to Lemma 2 the proof of Theorem 1 will be complete if we show

$$
\sup \int_{\overline{I_{M}}}\left|\tilde{\sigma}_{p}^{\alpha, *} a\right|^{p} d \mu<\infty
$$

where the supremum is taken over all $p$-atoms $a$. We may assume that $a$ is an arbitrary $p$-atom, with support $I, \mu(I)=2^{-M}$ and $I=I_{M}$. It is easy to see that $\sigma_{n}^{\alpha}(a)=0$, when $n \leq 2^{M}$. Therefore, we can suppose that $n>2^{M}$.

Let $x \in I_{M}$. Since $\|a\|_{\infty} \leq c 2^{M / p}$ we obtain

$$
\begin{gathered}
\left|\sigma_{n}^{\alpha} a(x)\right| \leq \int_{I_{M}}|a(t)|\left|K_{n}^{\alpha}(x+t)\right| d \mu(t) \\
\leq\|a(x)\|_{\infty} \int_{I_{M}}\left|K_{n}^{\alpha}(x+t)\right| d \mu(t) \\
\leq c_{\alpha} 2^{M / p} \int_{I_{M}}\left|K_{n}^{\alpha}(x+t)\right| d \mu(t) .
\end{gathered}
$$

Let $x \in I_{M}^{k, l}, 0 \leq k<l<M$. Then from Lemma 3 we get

$$
\left|\sigma_{n}^{\alpha} a(x)\right| \leq \frac{c_{\alpha, p} 2^{M(1 / p-1)} 2^{\alpha l+k}}{n^{\alpha}} .
$$

Let $x \in I_{M}^{k, M}, 0 \leq k<M$. Then from Lemma 3 we have

$$
\left|\sigma_{n}^{\alpha} a(x)\right| \leq c_{\alpha, p} 2^{M(1 / p-1)+k} .
$$


By combining (5), (13) and (14) we obtain

$$
\begin{aligned}
& \int \sup _{\overline{I_{M}}}\left|\frac{\sigma_{n}^{\alpha} a(x)}{n^{1 / p-1-\alpha}}\right|^{p} d \mu(x) \\
& =\sum_{k=0}^{M-2} \sum_{l=k+1}^{M-1} \sum_{x_{j}=0, j \in\{l+1, \ldots, M-1\}}^{1} \int_{I_{M}^{k, l}} \sup _{n>2^{M}}\left|\frac{\sigma_{n}^{\alpha} a(x)}{n^{1 / p-1-\alpha}}\right|^{p} d \mu(x) \\
& +\sum_{k=0}^{M-1} \int_{I_{M}^{k, M}} \sup _{n>2^{M}}\left|\frac{\sigma_{n}^{\alpha} a(x)}{n^{1 / p-1-\alpha}}\right|^{p} d \mu(x) \\
& \leq \frac{1}{2^{M(1-(1+\alpha) p)}} \sum_{k=0}^{M-2} \sum_{l=k+1}^{M-1} \sum_{x_{j}=0, j \in\{l+1, \ldots, N-1\}}^{1} \int_{I_{M}^{k, l}} \sup _{n>2^{M}}\left|\sigma_{n}^{\alpha} a(x)\right|^{p} d \mu(x) \\
& +\frac{1}{2^{M(1-(1+\alpha) p)}} \sum_{k=0}^{M-1} \int_{I_{M}^{k, M}} \sup _{n>2^{M}}\left|\sigma_{n}^{\alpha} a(x)\right|^{p} d \mu(x) \\
& \leq \frac{c_{\alpha, p}}{2^{M(1-(1+\alpha) p)}} \sum_{k=0}^{M-2} \sum_{l=k+1}^{M-1} \frac{1}{2^{l}} \frac{2^{M(1-p)} 2^{(\alpha l+k) p}}{2^{\alpha p M}} \\
& +\frac{c_{\alpha, p}}{2^{M(1-(1+\alpha) p)}} \frac{1}{2^{M}} \sum_{k=0}^{M-1} 2^{M(1-p)+p k} \\
& \leq \frac{c_{\alpha, p} 2^{\alpha p M}}{2^{\alpha p M}} \sum_{k=0}^{M-2} 2^{k p} \sum_{l=k+1}^{M-1} \frac{1}{2^{l(1-\alpha p)}} \\
& +\frac{c_{\alpha, p}}{2^{M(1-(1+\alpha) p)}} \sum_{k=0}^{M-1} \frac{2^{p k}}{2^{p M}} \leq c_{\alpha, p}<\infty .
\end{aligned}
$$

b) It is easy to show that under condition (11), there exists a sequence of positive integers $\left\{n_{k}, k \in \mathbb{N}_{+}\right\}$, such that

$$
\lim _{k \rightarrow \infty} \frac{\left(2^{2 n_{k}}+2\right)^{1 / p-1-\alpha}}{\varphi\left(2^{2 n_{k}}+2\right)}=\infty
$$

Let

$$
f_{n_{k}}=D_{2^{2 n_{k}+1}}-D_{2^{2 n_{k}}}
$$

It is evident

$$
\widehat{f}_{n_{k}}(i)=\left\{\begin{array}{l}
1, \text { if } i=2^{2 n_{k}}, \ldots, 2^{2 n_{k}+1}-1 \\
0, \text { otherwise. }
\end{array}\right.
$$

Then we can write

$$
S_{i} f_{n_{k}}= \begin{cases}D_{i}-D_{2^{2 n_{k}}}, & \text { if } i=2^{2 n_{k}}+1, \ldots, 2^{2 n_{k}+1}-1 \\ f_{n_{k}}, & \text { if } i \geq 2^{2 n_{k}+1} \\ 0, & \text { otherwise }\end{cases}
$$


From (6) we get

$$
\left\|f_{n_{k}}\right\|_{H_{p}}=\left\|f_{n_{k}}^{*}\right\|_{p}=\left\|D_{2^{2 n_{k}+1}}-D_{2^{2 n_{k}}}\right\|_{p} \leq c 2^{2 n_{k}(1-1 / p)} .
$$

Since $A_{0}^{\alpha-1}=1$, by (15) we can write

$$
\begin{gathered}
=\frac{\left|\sigma_{2^{2 n_{k}+1}}^{\alpha} f_{n_{k}}\right|}{\varphi\left(2^{2 n_{k}}+1\right)}\left|\sum_{j=1}^{2^{2 n_{k}+1}} A_{2^{2 n_{k}+1-j}}^{\alpha-1} S_{j} f_{n_{k}}\right| \\
=\frac{1}{\varphi\left(2^{2 n_{k}}+1\right) A_{2^{2 n_{k}+1}}^{\alpha}}\left|\sum_{j=2^{2 n_{k}+1}}^{2^{2 n_{k}+1}} A_{2^{2 n_{k}+1-j}}^{\alpha-1} S_{j} f_{n_{k}}\right| \\
=\frac{1}{\left.\varphi\left(2^{2 n_{k}}+1\right) A_{2^{2 n_{k}}+1}^{\alpha}+1\right) A_{2^{2 n_{k}}+1}^{\alpha}}\left|A_{0}^{\alpha-1}\left(D_{2^{2 n_{k}+1}}-D_{2^{2 n_{k}}}\right)\right| \\
=\frac{1}{\varphi\left(2^{\left.2 n_{k}+1\right) A_{2^{2 n_{k}+1}}^{\alpha}}\left|A_{0}^{\alpha-1} w_{2^{2 n_{k}}}\right|\right.} \\
\geq \frac{c}{\varphi\left(2^{2 n_{k}}+1\right)\left(2^{2 n_{k}}+1\right)^{\alpha}} .
\end{gathered}
$$

From (16) we have

$$
\begin{aligned}
& \frac{c /\left(\varphi\left(2^{2 n_{k}}+1\right)\left(2^{2 n_{k}}+1\right)^{\alpha}\right) \mu\left\{x:\left|\tilde{\sigma}^{\alpha, *} f\right| \geq c /\left(\varphi\left(2^{2 n_{k}}+1\right)\left(2^{2 n_{k}}+1\right)^{\alpha}\right)\right\}^{1 / p}}{\left\|f_{n_{k}}\right\|_{H_{p}}} \\
& \geq \frac{c}{\varphi\left(2^{2 n_{k}}+1\right)\left(2^{2 n_{k}}+1\right)^{\alpha}} \frac{1}{2^{2 n_{k}(1-1 / p)}} \geq \frac{c\left(2^{2 n_{k}}+1\right)^{1 / p-1-\alpha}}{\varphi\left(2^{2 n_{k}}+1\right)} \rightarrow \infty, \text { as } k \rightarrow \infty .
\end{aligned}
$$

Theorem 1 is proven.

Proof of Theorem 2. Suppose that

$$
\sum_{m=1}^{\infty} \frac{\left\|\sigma_{m}^{\alpha} F\right\|_{p}^{p}}{m^{2-(1+\alpha) p}} \leq\|F\|_{H_{p}}^{p} .
$$

Then by using (10) we have

$$
\begin{gathered}
\sum_{m=1}^{\infty} \frac{\left\|\sigma_{m}^{\alpha} F\right\|_{H_{p}}^{p}}{m^{2-(1+\alpha) p}} \\
=\sum_{m=1}^{\infty} \frac{\int_{G}\left\|\widetilde{\sigma_{m}^{\alpha} F^{(t)}}\right\|_{p}^{p} d t}{m^{2-(1+\alpha) p}} \leq \int_{G} \sum_{m=1}^{n} \frac{\left\|\sigma_{m}^{\alpha} \widetilde{F^{(t)}}\right\|_{p}^{p}}{m^{2-(1+\alpha) p}} d t \\
\leq \int_{G}\left\|\widetilde{F^{(t)}}\right\|_{H_{p}}^{p} d t \sim \int_{G}\|F\|_{H_{p}}^{p} d t=\|F\|_{H_{p}}^{p} .
\end{gathered}
$$


According to Theorem 1 and (17) the proof of Theorem 2 will be complete, if we show

$$
\sum_{m=1}^{\infty} \frac{\left\|\sigma_{m}^{\alpha} a\right\|_{p}^{p}}{m^{2-(1+\alpha) p}} \leq c_{\alpha}<\infty,
$$

for every $p$-atom $a$. Analogously to first part of Theorem 1 we can assume that $n>2^{M}$ and $a$ be an arbitrary $p$-atom, with support $I, \mu(I)=2^{-M}$ and $I=I_{M}$.

Let $x \in I_{M}$. Since $\sigma_{n}$ is bounded from $L_{\infty}$ to $L_{\infty}$ (the boundedness follows from (8) ) and $\|a\|_{\infty} \leq c 2^{M / p}$ we obtain

$$
\begin{gathered}
\int_{I_{M}}\left|\sigma_{m}^{\alpha} a\right|^{p} d \mu \leq \int_{I_{M}}\left\|K_{m}^{\alpha}\right\|_{1}^{p}\|a\|_{\infty}^{p} d \mu \\
\leq c_{\alpha, p} \int_{I_{M}}\|a\|_{\infty}^{p} d \mu \leq c_{\alpha, p}<\infty .
\end{gathered}
$$

Hence

$$
\begin{gathered}
\sum_{m=2^{M}+1}^{\infty} \frac{\int_{I_{M}}\left|\sigma_{m}^{\alpha} a\right|^{p} d \mu}{m^{2-(1+\alpha) p}} \\
\leq c_{\alpha, p} \sum_{m=2^{M}+1}^{\infty} \frac{1}{m^{2-(1+\alpha) p}} \\
\leq \frac{c_{\alpha, p}}{2^{M(1-(1+\alpha) p)}} \leq c_{\alpha, p}<\infty .
\end{gathered}
$$

By combining (5), (13) and (14) analogously to first part of Theorem 1 we can write

$$
\begin{gathered}
\sum_{m=2^{M}+1}^{\infty} \frac{\int_{I_{M}}\left|\sigma_{m}^{\alpha} a\right|^{p} d \mu}{m^{2-(1+\alpha) p}} \\
=\sum_{m=2^{M}+1}^{\infty}\left(\sum_{k=0}^{M-2} \sum_{l=k+1}^{M-1} \sum_{x_{j}=0, j \in\{l+1, \ldots, M-1\}}^{1} \frac{\int_{I_{M}^{k, l}}\left|\sigma_{m}^{\alpha} a\right|^{p} d \mu}{m^{2-(1+\alpha) p}}+\sum_{k=0}^{M-1} \frac{\int_{I_{M}^{k, M}}\left|\sigma_{m}^{\alpha} a\right|^{p} d \mu}{m^{2-(1+\alpha) p}}\right) \\
\leq \sum_{m=2^{M}+1}^{\infty}\left(\frac{c_{\alpha, p} 2^{M(1-p)}}{m^{2-p}} \sum_{k=0}^{M-2} \sum_{l=k+1}^{M-1} \frac{2^{p(\alpha l+k)}}{2^{l}}+\frac{c_{\alpha, p} 2^{M(1-p)}}{m^{2-(1+\alpha) p}} \sum_{k=0}^{M-1} \frac{2^{p k}}{2^{M}}\right) \\
<c_{\alpha, p} 2^{M(1-p)} \sum_{m=2^{M}+1}^{\infty} \frac{1}{m^{2-p}}+c_{\alpha, p} \sum_{m=2^{M}+1}^{\infty} \frac{1}{m^{2-(1+\alpha) p}} \leq c_{\alpha, p}<\infty,
\end{gathered}
$$

which completes the proof of Theorem 2 


\section{REFERENCES}

[1] I. Blahota, On a norm inequality with respect to Vilenkin-like systems, Acta Math. Hungar. 89 (2000), no. 1-2, 15-27.

[2] I. Blahota, and G. Tephnadze, On the $(C, \alpha)$-means with respect to the Walsh system, to appear in Analysis Mathematica.

[3] I. Blahota, and G. Tephnadze, Strong convergence theorem for Vilenkin-Fejér means, to appear in Publicationes Mathematicae Debrecen.

[4] I. Blahota, G. Gát, and U. Goginava, Maximal operators of Fejér means of VilenkinFourier series, J. Inequal. Pure Appl. Math. 7 (2006), no. 4, Article 149, 7 pp. (electronic).

[5] N. J. Fujii, A maximal inequality for $H^{1}$-functions on a generalized Walsh-Paley group, Proc. Amer. Math. Soc. 77 (1979), no. 1, 111-116.

[6] G. Gát, Investigations of certain operators with respect to the Vilenkin system, Acta Math. Hungar. 61 (1993), no. 1-2, 131-149.

[7] G. Gát, U. Goginava, A weak type inequality for the maximal operator of $(C, \alpha)$-means of Fourier series with respect to the Walsh-Kaczmarz system, Acta Math. Hungar. 125 (2009), no. 1-2, 65-83.

[8] U. Goginava, Maximal operators of Fejér means of double Walsh-Fourier series, Acta Math. Hungar. 115 (2007), no. 4, 333-340.

[9] U. Goginava, The maximal operator of the $(C, \alpha)$ means of the Walsh-Fourier series, Ann. Univ. Sci. Budapest. Sect. Comput. 26 (2006), 127-135.

[10] U. Goginava, On the approximation properties of Cesàro means of negative order of Walsh-Fourier series, J. Approx. Theory 115 (2002), no. 1, 9-20.

[11] B. Golubov, A. Efimov and V. Skvortsov, Walsh series and transformations, Dordrecht, Boston, London, 1991. Kluwer Acad. publ, 1991.

[12] K. Nagy, Approximation by Cesàro means of negative order of Walsh-KaczmarzFourier series, East J. Approx. 16 (2010), no. 3, 297-311.

[13] J. Pál and P. Simon, On a generalization of the concept of derivative, Acta Math. Acad. Sci. Hungar. 29 (1977), no. 1-2, 155-164.

[14] F. Schipp, Certain rearrangements of series in the Walsh system, (Russian) Mat. Zametki 18 (1975), no. 2, 193-201.

[15] F. Schipp, W.R. Wade, P. Simon and J. Pál, Walsh series, An Introduction to Dyadic Harmonic Analysis, Akadémiai Kiadó, (Budapest-Adam Hilger (Bristol-New-York)), 1990.

[16] P. Simon, Strong convergence theorem for Vilenkin-Fourier series, J. Math. Anal. Appl. 245 (2000), no. 1, 52-68.

[17] P. Simon, Cesaro summability with respect to two-parameter Walsh systems, Monatsh. Math. 131 (2000), no. 4, 321-334.

[18] P. Simon, Investigations with respect to the Vilenkin system, Ann. Univ. Sci. Budapest. Eötvös Sect. Math. 27 (1984), 87-101 (1985).

[19] P. Simon, Strong convergence of certain means with respect to the Walsh-Fourier series, Acta Math. Hungar. 49 (1987), no. 3-4, 425-431.

[20] P. Simon and F. Weisz, Weak inequalities for Cesàro and Riesz summability of WalshFourier series, J. Approx. Theory 151 (2008), no. 1, 1-19.

[21] B. Smith, A strong convergence theorem for $H^{1}(T)$. Banach spaces, harmonic analysis, and probability theory, 169-173, Lecture Notes in Math., 995, Springer, BerlinNew York, 1983.

[22] G. Tephnadze, Fejér means of Vilenkin-Fourier series, Studia Sci. Math. Hungar. 49 (2012), no. 1, 79-90.

[23] G. Tephnadze, On the maximal operators of Vilenkin-Fejér means, Turkish J. Math. 37 (2013), no. 2, 308-318. 
[24] G. Tephnadze, On the maximal operators of Vilenkin-Fejér means on Hardy spaces, Math. Inequal. Appl. 16 (2013), no. 1, 301-312.

[25] G. Tephnadze, A note on the Fourier coefficients and partial sums of Vilenkin-Fourier series, Acta Math. Acad. Paedagog. Nyházi. (N.S.) 28 (2012), no. 2, 167-176.

[26] G. Tephnadze, Strong convergence theorems for Walsh-Fejér means, Acta Math. Hungar. 142 (2014), no. 1, 244-259.

[27] G. Tephnadze, On the partial sums of Vilenkin-Fourier series, Journal of Contemporary Mathematical Analysis, Vol. 49, No. 1, 2014, 23-32.

[28] N. Ya. Vilenkin, On a class of complete orthonormal systems, Amer. Math. Soc. Transl. (2) 28 (1963), 1-35.

[29] F. Weisz, Martingale Hardy spaces and their applications in Fourier analysis, Lecture Notes in Math. 1568, Springer, Berlin, 1568, Springer-Verlag, Berlin, 1994.

[30] F. Weisz, Cesàro summability of one- and two-dimensional Walsh-Fourier series, Anal. Math. 22 (1996), no. 3, 229-242.

[31] F. Weisz, Weak type inequalities for the Walsh and bounded Ciesielski systems, Anal. Math. 30 (2004), no. 2, 147-160.

[32] F. Weisz, Hardy spaces and Cesàro means of two-dimensional Fourier series, Approximation theory and function series (Budapest, 1995), 353-367, Bolyai Soc. Math. Stud., 5, János Bolyai Math. Soc., Budapest, 1996.

[33] F. Weisz, $(C, \alpha)$ summability of Walsh-Fourier series, Anal. Math. 27 (2001), no. 2, 141-155.

[34] F. Weisz, Strong convergence theorems for two-parameter Walsh-Fourier and trigonometric-Fourier series, Studia Math. 117 (1996), no. 2, 173-194.

[35] F. Weisz, Summability of multi-dimensional Fourier series and Hardy spaces, Mathematics and its Applications, 541. Kluwer Academic Publishers, Dordrecht, 2002.

I. Blahota, Institute of Mathematics and Computer Sciences, College of Nyíregyháza, P.O. Box 166, Nyíregyháza, H-4400, Hungary

E-mail address: blahota@nyf.hu

G. Tephnadze, Department of Mathematics, Faculty of Exact and Natural Sciences, Tbilisi State University, Chavchavadze str. 1, Tbilisi 0128, Georgia and Department of Engineering Sciences and Mathematics, Luleß University of Technology, SE-971 87 Lule $\AA$, Sweden

E-mail address: giorgitephnadze@gmail.com

R. Toledo, Institute of Mathematics and Computer Sciences, College of NyíregyházA, P.O. Box 166, Nyíregyháza, H-4400, Hungary

E-mail address: toledo@nyf.hu 